$$
\lambda_{2 n-1}>2 \text { (first hypothesis), }
$$

or

$$
\lambda_{2 n-1}>1+(K+1)^{-1} \text { (second hypothesis). }
$$

Hence, we have infinitely many solutions of (1) with $c=2$ or $c=1$ $+(K+1)^{-1}$.

\title{
REFERENCES
}

1. B. Segre, Lattice points in infinite domains, and asymmetric Diophantine approximations, Duke Math. J. vol. 12 (1945) pp. 337-365.

2. R. M. Robinson, Unsymmetrical approximation of irrational numbers, Bull. Amer. Math. Soc. vol. 53 (1947) pp. 351-361.

3. - The approximation of irrational numbers by fractions with odd or even terms, Duke Math. J. vol. 7 (1940) pp. 354-359.

University of Oregon

\section{A NOTE ON SUBSERIES CONVERGENCE}

\section{CHARLES W. MCARTHUR ${ }^{1}$}

1. Introduction. Throughout this paper $X$ will denote a Banach space ( $B$-space), $X^{*}$ its normed conjugate space, and $\sum_{i=1}^{\infty} x_{i}$ a series in $X$. A series $\sum_{i=1}^{i} x_{i}$ is subseries convergent if and only if

(A) Each subseries of $\sum_{i=1}^{\infty} x_{i}$ converges. Additional conditions, (B) through $(\mathrm{H})$, are introduced below. The main purpose of this note is to give new and simple proofs of the equivalence of each of $(B),(G)$, and $(\mathrm{H})$ to $(A)$.

The equivalence of (A) and (B), the Orlicz-Pettis theorem, was first stated and proved by Orlicz [11, Satz 2] for weakly complete $X$. It was stated by Banach [2, p. 240] for general $X$. The first proof of the equivalence of (A) and (B) for $X$ a $B$-space was given by Pettis [12, Theorem 2.32]. Conditions $(\mathrm{G})$ and $(\mathrm{H})$ were suggested by Gelfand $[8]$ as definitions of "strong unconditional convergence" of $\sum_{i=1}^{\infty} x_{i}$ and he $[8$, Satz $5 \mathrm{a}$, p. 245] stated their equivalence. The equivalence of $(\mathrm{H})$ and $(\mathrm{A})$ is shown in [9]. Thus, the equivalence of $(A),(B),(G)$ and $(\mathrm{H})$ is well known.

Presented to the Society, November 18, 1960; received by the editors September $13,1960$.

1 This work was supported by a Summer Research Grant from the Research Council of the Florida State University. 
Dunford [6, Theorem 32] has shown that (C) (as well as (A) and (H)) follow from (B). However, condition (C) and its obviously equivalent forms (D) and (E) seem not to have been recognized before as equivalent to subseries convergence. Theorem 1 provides a new proof of the Orlicz-Pettis theorem. Corollary 2 is due to Dunford [6, Theorem 75]. Its proof is different from his. Gelfand's proof of $(\mathrm{H}) \Rightarrow(\mathrm{G})$ is defective due to his use of an incorrect criterion for compactness of a subset of a $B$-space (see [13, Corollary 3.2]). Also he omits the proof of $(\mathrm{G}) \Rightarrow(\mathrm{H})$. Theorem 2 includes a proof of the equivalence of $(\mathrm{G})$ and $(\mathrm{H})$.

In addition to the proofs in $[11 ; 12 ; 6]$ of the Orlicz-Pettis theorem different proofs have been given in $[1$, p. $24 ; 3$, Corollary $9 ; 5$, p. 60]. A detailed exposition of Dunford's proof is given in [10, pp. 61-62].

In the sequel the $B$-space of real bounded sequences $a=\left\{a_{i}\right\}$ with $\|a\|=\sup _{i}\left|a_{i}\right|$ will be denoted by $(m)$ and the $B$-space of real sequences $a=\left\{a_{i}\right\}$ such that $\sum_{i=1}^{\infty}\left|a_{i}\right|<+\infty$ with $\|a\|=\sum_{i=1}^{\infty}\left|a_{i}\right|$ will be represented by $(l)$.

In the statements of conditions (B) through $(G)$ which follow the closed linear manifold generated by the sequence $\left\{x_{i}\right\}$ will be denoted by $\left[x_{i}\right]$. Also for a fixed series $\sum_{i=1}^{\infty} x_{i}$ and $K$ a subset of $(m)$ we define

$$
S(K)=\left\{\sum_{i \in \sigma} k_{i} x_{i}:\left\{k_{i}\right\} \in K \text { and } \sigma \text { finite }\right\} .
$$

(B) Each subseries of $\sum_{i=1}^{\infty} x_{i}$ converges weakly to an element, i.e., corresponding to each increasing sequence $\sigma=\left\{n_{i}\right\}$ of positive integers there exists $x_{\sigma} \in X$ such that $f\left(x_{\sigma}\right)=\sum_{i=1}^{\infty} f\left(x_{n_{i}}\right)$ for all $f \in X^{*}$.

(C) If $\left\{f_{n}\right\}$ is a sequence in $X^{*}$ such that $f_{n}(x) \rightarrow 0$ for each $x \in\left[x_{i}\right]$ then $\lim _{n \rightarrow \infty} \sum_{i=1}^{\infty}\left|f_{n}\left(x_{i}\right)\right|=0$.

(D) If $\left\{f_{n}\right\}$ is a sequence in $X^{*}$ such that $f_{n}(x) \rightarrow 0$ for each $x \in\left[x_{i}\right]$ and $K$ is a bounded subset of $(m)$ then $\lim _{n \rightarrow \infty} f_{n}(x)=0$ uniformly for $x \in S(K)$.

(E) If $\left\{f_{n}\right\}$ is a sequence in $X^{*}$ such that $f_{n}(x) \rightarrow 0$ for each $x \in\left[x_{i}\right]$ then $\lim _{n \rightarrow \infty} f_{n}(x)=0$ uniformly for $x \in\left\{\sum_{i \in \sigma} x_{i}: \sigma\right.$ finite $\}$. $(m)$.

(F) $S(K)$ is conditionally compact for each bounded subset $K$ of

(G) $\left\{\sum_{i \in \sigma} x_{i}: \sigma\right.$ finite $\}$ is conditionally compact.

(H) $\lim _{n \rightarrow \infty} \sum_{i=n}^{\infty}\left|f\left(x_{i}\right)\right|=0$ uniformly for $f \in X^{*}$ such that $\|f\| \leqq 1$.

2. The theorems. Gelfand [8, p. 268] and Phillips [13, Corollary 3.2] have shown that $a$ subset $M$ of $a$ separable $B$-space $X$ is conditionally compact if and only if $\lim _{n \rightarrow \infty} f_{n}(x)=0$ uniformly for $x \in M$ when- 
ever $\lim _{n \rightarrow \infty} f_{n}(x)=0$ for all $x \in X$. We state the following simple extension of their result.

Lemma 1. If $X_{0}$ is a closed separable subspace of an arbitrary B-space $X$ then a subset $M$ of $X_{0}$ is conditionally compact in $X$ if and only if $\lim _{n \rightarrow \infty} f_{n}(x)=0$ uniformly for $x \in M$ whenever $\left\{f_{n}\right\}$ is a sequence in $X^{*}$ such that $\lim _{n \rightarrow \infty} f_{n}(x)=0$ for all $x \in X_{0}$.

THEOREM 1.

$$
(\mathrm{A}) \Rightarrow(\mathrm{B}) \Rightarrow(\mathrm{C}) \Rightarrow(\mathrm{D}) \Rightarrow(\mathrm{E}) \Leftrightarrow(\mathrm{G}) \Rightarrow(\mathrm{A}) .
$$

Also $(\mathrm{D}) \Leftrightarrow(\mathrm{F})$. Hence, (A) through $(\mathrm{G})$ are equivalent.

Proof. That (A) implies (B) is obvious. Now assume (B). For each $f \in X^{*}$ the real series $\sum_{i=1}^{\infty} f\left(x_{i}\right)$ is subseries convergent so it follows that the sequence $\left\{f\left(x_{i}\right)\right\}$ is an element of $(l)$. Let $\left\{f_{n}\right\}$ be a sequence in $X^{*}$ satisfying the hypothesis of (C). Then, for each $n$, $\lambda_{n} \equiv\left\{f_{n}\left(x_{i}\right)\right\} \in(l)$. We wish to show that $\left\{\lambda_{n}\right\}$ converges to zero in $(l)$. To do this it suffices [2, pp. 137-139] to show that $\lim _{n \rightarrow \infty} \sum_{i=1}^{\infty} \epsilon_{i} f_{n}\left(x_{i}\right)$ $=0$ for each real sequence $\left\{\epsilon_{i}\right\}$ with $\epsilon_{i}$ one of the values $1,-1$, or 0 for each $i$. Let $\left\{\epsilon_{i}\right\}$ denote such a sequence. Let $\sigma_{+}=\left\{i: \epsilon_{i} \geqq 0\right\}$ and $\sigma_{-}=\left\{i: \epsilon_{i}<0\right\}$. By (B), there exist $x_{\sigma+}$ and $x_{\sigma_{-}}$in $X$ such that

$$
f\left(x_{\sigma+}\right)=\sum_{i \in \sigma+} f\left(x_{i}\right) \quad \text { and } f\left(x_{\sigma-}\right)=\sum_{i \in \sigma-} f\left(x_{i}\right)
$$

for all $f \in X^{*}$. Indeed, $x_{\sigma+}$ and $x_{\sigma-}$ are elements of $\left[x_{i}\right]$. Suppose, e.g., that $x_{\sigma_{+}} \notin\left[x_{i}\right]$. Then there would exist $f \in X^{*}$ such that $f\left(x_{\sigma+}\right) \neq 0$ and $f(x)=0$ for all $x \in\left[x_{i}\right]$. This gives the contradiction $f\left(x_{\sigma+}\right)$ $=\sum_{i \in \sigma+} f\left(x_{i}\right)=0$. Hence, it follows that

$$
\sum_{i=1}^{\infty} \epsilon_{i} f_{n}\left(x_{i}\right)=\sum_{i \in \sigma+} f_{n}\left(x_{i}\right)-\sum_{i \in \sigma-} f_{n}\left(x_{i}\right)=f_{n}\left(x_{\sigma+}\right)-f_{n}\left(x_{\sigma-}\right)
$$

and $\left(f_{n}\left(x_{\sigma+}\right)-f_{n}\left(x_{\sigma-}\right)\right) \rightarrow 0$ by the hypothesis of (C). Thus, $\left\|\lambda_{n}\right\|_{(l)} \rightarrow 0$, i.e., $\lim _{n \rightarrow \infty} \sum_{i=1}^{\infty}\left|f_{n}\left(x_{i}\right)\right|=0$. This proves that $(\mathrm{B}) \Rightarrow(\mathrm{C})$. That $(\mathrm{C}) \Rightarrow(\mathrm{D})$ and $(\mathrm{D}) \Rightarrow(\mathrm{E})$ are clear.

Taking $X_{0}=\left[x_{i}\right]$ and $M=\left\{\sum_{i \in \sigma} x_{i}: \sigma\right.$ finite $\},(\mathrm{E})$ and (G) are equivalent by Lemma 1 . Similarly (D) and (F) are equivalent by Lemma 1.

It remains to show that $(G) \Rightarrow(A)$. Hence, assume $(G)$, i.e., $M \equiv\left\{\sum_{i \in \sigma} x_{i}: \sigma\right.$ finite $\}$ is conditionally compact. Thus $M$ is bounded from which it readily follows that $\sum_{i=1}^{\infty}\left|f\left(x_{i}\right)\right|<+\infty$ for each $f \in X^{*}$. Now suppose there exists a subseries $\sum_{i=1}^{\infty} x_{k_{i}}$ which does not converge. Then there exists $\epsilon_{0}>0$ and an increasing sequence of indices $\left\{p_{n}\right\}$ such that 


$$
\left\|\sum_{i=p_{n}+1}^{p_{n+1}} x_{k_{i}}\right\| \geqq \epsilon_{0} \quad \text { for each } n
$$

For each $n$, let

$$
s_{n}=\sum_{i=p_{n}+1}^{p_{n}+1} x_{k_{i}}
$$

It follows, since

$$
\sum_{i=1}^{\infty}\left|f\left(x_{k_{i}}\right)\right|<+\infty \text { for each } f \in X^{*},
$$

that

$$
\lim _{n \rightarrow \infty} f\left(s_{n}\right)=0 \quad \text { for each } f \in X^{*} .
$$

Furthermore, since $M$ is conditionally compact, there exists a subsequence $\left\{s_{n_{m}}\right\}$ which converges. Let $x=\lim _{m \rightarrow \infty} s_{n_{m}}$. From (1) it follows that $\|x\| \geqq \epsilon_{0}>0$. This is incompatible with (2), which implies that $\|x\|=0$. Thus it follows that $(\mathrm{G}) \Rightarrow(\mathrm{A})$.

Corollary 1. A series $\sum_{i=1}^{\infty} x_{i}$ in $X$ satisfies condition (B) if and only if for each $a=\left\{a_{i}\right\} \in(m)$, the series $\sum_{i=1}^{\infty} a_{i} x_{i}$ is subseries convergent.

Proof. Suppose that $\sum_{i=1}^{\infty} x_{i}$ satisfies (B) and $a=\left\{a_{i}\right\} \in(m)$. By Theorem 1, (D) holds so if $\left\{f_{n}\right\}$ is a sequence in $X^{*}$ such that $f_{n}(x) \rightarrow 0$ on $\left[x_{i}\right]$ then $\lim _{n \rightarrow \infty} f_{n}(x)=0$ uniformly for $x \in\left\{\sum_{i \in \sigma} a_{i} x_{i}: \sigma\right.$ is finite $\}$. But $\left[x_{i}\right]=\left[a_{i} x_{i}\right]$ so condition (E) holds for the series $\sum_{i=1}^{\infty} a_{i} x_{i}$. Thus, by Theorem 1, (A) follows for the series $\sum_{i=1}^{\infty} a_{i} x_{i}$. The converse implication is trivial.

COROLlaRY 2. If each subseries of $\sum_{i=1}^{\infty} x_{i}$ is weakly convergent to an element of $X$ then for each $a=\left\{a_{i}\right\} \in(m)$ the series $\sum_{i=1}^{\infty} a_{i} x_{i}$ is subseries convergent and the linear operator $L$ defined from $(m)$ to $X$ by

$$
L(a)=\sum_{i=1}^{\infty} a_{i} x_{i} \quad \text { for each } a \in(m)
$$

is compact. Furthermore, the linear operator defined from $X^{*}$ to $(l)$ by

$$
T(f)=\left\{f\left(x_{i}\right)\right\} \quad \text { for each } f \in X^{*}
$$

is compact. Hence,

$$
\lim _{n \rightarrow \infty} \sum_{i=n}^{\infty}\left|f\left(x_{i}\right)\right|=0 \text { uniformly for }\|f\| \leqq 1 .
$$


Proof. By Corollary $1, L$ is well defined and it is clear that $L$ is linear. Moreover $L$ is compact because if $K$ denotes the unit sphere in $(m)$ then $L(K) \subset \mathrm{Cl} S(K)$ where $S(K)$ is conditionally compact by (F). Thus, $L$ is compact. Denoting the adjoint of $T$ by $T^{*}$ it is readily seen that $L=T^{*}$ so $T$ is compact since $[7$, p. 485] a linear operator from one $B$-space to another is compact if and only if its adjoint operator is. The last statement of the theorem is the result of a well known criterion $[4$, p. 691$]$ for conditional compactness in $(l)$ applied to the set $\left\{\left\{f\left(x_{i}\right)\right\}:\|f\| \leqq 1\right\}$.

Theorem 2 (Summary). Conditions (A) through $(\mathrm{H})$ are equivalent. Moreover, the operators $L$ and $T$ (of Corollary 2) are compact if and only if one, and hence all, of conditions (A) through (H) holds.

PRoof. In the proof of Corollary 2 it was noted that $L$ was compact if and only if $T$ was and that $T$ was compact if and only if $(\mathrm{H})$ held. Because of this and Theorem 1 only $(\mathrm{H}) \Rightarrow(\mathrm{A})$ remains to be shown. An elegant proof of the equivalence of $(\mathrm{H})$ and $(\mathrm{A})$ via MooreSmith convergence is given in [9]. We include a proof of $(\mathrm{H}) \Rightarrow(\mathrm{A})$ for completeness. Assume $(\mathrm{H})$ and let $\epsilon>0$ be given. There then exists $n_{\mathrm{e}}$ such that

$$
\sum_{i=n_{\epsilon}}^{\infty}\left|f\left(x_{i}\right)\right|<\epsilon \quad \text { if }\|f\| \leqq 1 .
$$

Hence, if $\sigma$ is a finite set of positive integers disjoint from $\left[1, n_{\epsilon}\right]$ then

$$
\left\|\sum_{i \in \sigma} x_{i}\right\|=\sup _{\|f\| \leqq 1}\left|f\left(\sum_{i \in \sigma} x_{i}\right)\right| \leqq \sum_{i=n_{\epsilon}}^{\infty}\left|f\left(x_{i}\right)\right|<\epsilon .
$$

From this it is evident that the partial sums of each subseries of $\sum_{i=1}^{\infty} x_{i}$ form a Cauchy sequence and hence converge since $X$ is complete.

It is known that if a series in a Banach space is subseries convergent then each of its rearrangements is subseries convergent. This fact is an immediate corollary of Theorem 1 since it is clear that for each permutation $\tau$ of the positive integers the sets

$$
\left\{\sum_{i \in \sigma} x_{\tau(i)}: \sigma \text { finite }\right\} \text { and }\left\{\sum_{i \in \sigma} x_{i}: \sigma \text { finite }\right\}
$$

are equal.

\section{REFERENCES}

1. A. Alexiewicz, On sequences of operations. I, Studia Math. vol. 11 (1950) pp. 1-30. 
2. S. Banach, Théorie des opérations linéaires, Warsaw, Monogr. mat. vol. 1, 1932.

3. C. Bessaga and A. Pełczynski, On bases and unconditional convergence of series in Banach space, Studia Math. vol. 17 (1958) pp. 151-164.

4. L. W. Cohen and N. Dunford, Transformations on sequence spaces, Duke Math. J. vol. 3 (1937) pp. 689-701.

5. M. M. Day, Normed linear spaces, Ergebnisse der Mathematik, BerlinGöttingen-Heidelberg, Springer-Verlag, 1958.

6. N. Dunford, Uniformity in linear spaces, Trans. Amer. Math. Soc. vol. 44 (1938) pp. 305-356.

7. N. Dunford and J. T. Schwartz, Linear operators. Part I, New York, Interscience Publishers, Inc., 1958.

8. I. M. Gelfand, Abstrakte Funktionen und lineare Operatoren, Mat. Sb. (N. S.) (4) vol. 46 (1938) pp. 235-286.

9. T. H. Hildebrandt, On unconditional convergence in normed vector spaces, Bull. Amer. Math. Soc. vol. 46 (1940) pp. 959-962.

10. E. Hille and R. S. Phillips, Functional analysis and semigroups, Amer. Math. Soc. Colloquium Publications, vol. 31, 1957.

11. W. Orlicz, Beiträge zur Theorie der Orthogonalentwicklungen. II, Studia Math. vol. 1 (1929) pp. 241-255.

12. B. J. Pettis, On integration in vector spaces, Trans. Amer. Math. Soc. vol. 44 (1938) pp. 277-304.

13. R. S. Phillips, On linear transformations, Trans. Amer. Math. Soc. vol. 48 (1940) pp. 516-541.

Florida State University 